\title{
Clinical Supportive and Palliative Care
}

\section{P 009 THE PERSPECTIVES OF BEREAVED RELATIVES AND CLINCIANS ABOUT SEDATION IN END OF LIFE CARE: INSIGHTS FROM QUALITATIVE CASE STUDIES}

Jane Seymour, ${ }^{1}$ Jayne Brown'. ${ }^{1}$ The University of Nottingham, Nottingham, GB; ${ }^{2}$ De Montfort University, Leciester, GB

10.1136/bmjspcare-2014-000654.50

Background There are few studies which compare the experiences of clinical staff and bereaved relatives about sedation use in end of life care.

Aim To report aspects of the UNBIASED study (UK, Netherlands and Belgium International Sedation Study), comparing how UK clinicians and bereaved relatives recall the use of sedation in end of life care.

Methods Qualitative case study design using face to face interviews. Setting: Hospitals, the domestic home, and hospices. Participants: doctors, nurses and bereaved relatives involved in care of cancer patients who received continuous sedation until death. Senior clinical staff identified eligible decedents and then nominated the physicians and nurses most involved in their care; they then used their discretion to invite bereaved relatives to take part, at least three months after the death.

Findings 22 case studies were completed in the UK arm of the project. Seven cases were complete, in that they had perspectives from at least one nurse $(n=10)$, one doctor $(n=9)$ and one relative $(n=7)$. This presentation focuses on these 7 cases. Relatives recalled that gaining control of some patients' symptoms was difficult to achieve and they regretted the time taken to gain this. Clinicians reported that the movement towards continuous sedation unfolded over time with no particular 'moment' of 
decision making. Careful use of medications to control difficult symptoms without making the patient completely unconsciousness was seen as the ideal by both relatives and clinicians. Some relatives and clinicians were concerned about hastening death; some relatives regretted that this was not possible in UK practice. Relatives expressed a range of understandings about how sedative medications worked. Understanding patients' wishes was important for both relatives and clinicians.

Conclusion Detailed case studies can illuminate difficult areas of practice and provide pointers for practice development. Implications for communication and decision-making practice will be explored. 